\title{
An analysis of diagnostic practices in a mammography unit in a tertiary hospital in South Africa
}

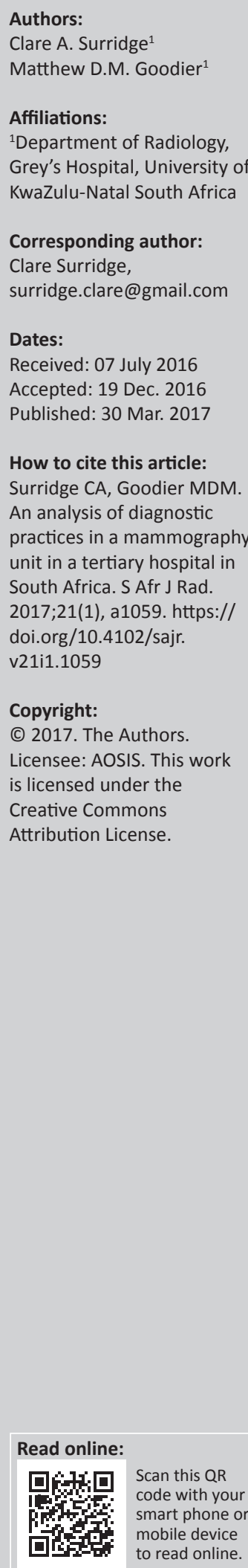

Background: Breast cancer is the most common cancer in females in South Africa. The reporting of breast imaging has been standardised internationally using the Breast Imaging and Reporting Data System (BI-RADS), which includes guidelines for reporting of breast lesions and further management. Ultrasound-guided core-needle breast (UGCNB) biopsy is a widely used method of obtaining histological diagnoses of breast lesions to assist with planning definitive management.

Objectives: To perform an audit of the UGCNB biopsies performed at the Grey's Hospital Mammography Department and assess the accuracy of the radiologists' use of the BI-RADS scoring system.

Methods: Records of all patients who underwent UGCNB biopsy between 01 January 2014 and 31 October 2015 were reviewed. A retrospective study was performed.

Results: A total of 304 UGCNB biopsies were performed on 291 patients. The mean age was 49.2 (s.d. = 15.9) years. Tissue samples from 303 lesions were adequate for histological assessment, and of these, $51 \%$ of the lesions were malignant whilst $49 \%$ were benign. The most common malignant and benign diagnoses were invasive ductal carcinoma and fibroadenoma respectively. The BI-RADS scoring of the radiologists demonstrated a positive predictive value of $61.6 \%$ for the identification of possible malignant lesions.

Conclusion: This study describes the patient and lesion profile and unit practices in a tertiary hospital setting in South Africa. The radiologists' application of the BI-RADS scoring largely conforms to the BI-RADS guidelines. The study highlights several challenges encountered by a breast imaging programme in an under-resourced setting as well as making recommendations in overcoming these challenges.

\section{Introduction}

Breast cancer is the most common cancer found in women in South Africa and causes significant mortality and morbidity. ${ }^{1}$ Early diagnosis, allowing the initiation of correct management, is vital for combatting the disease and reducing mortality. It is established practice that patients presenting with breast masses are assessed with a triple approach comprising clinical, radiological and histological evaluations. The radiologist thus plays an important role along with the surgeon and the pathologist in the evaluation and management of such patients. Reporting and further management of breast lesions have been standardised by the American College of Radiology by means of the Breast Imaging Reporting and Data System (BI-RADS) guidelines. ${ }^{2}$ These guidelines have become a widely accepted risk assessment and quality assurance tool in breast imaging.

BI-RADS guidelines exist for mammography, ultrasound and magnetic resonance imaging of the breasts. The BI-RADS assessment categorisation guides patient management and is based on the most abnormal finding of all imaging modalities used to assess the patient. ${ }^{2}$ The final summary assessment scores the lesion(s) from 0 to 6 . Category 0 is an incomplete investigation. Category 1 is a negative investigation. Category 2 describes benign findings. Category 3 lesions are probably benign, with a less than $2 \%$ chance of malignancy. Category 4 lesions are suspicious lesions and are subcategorised into $\mathrm{A}, \mathrm{B}$ and $\mathrm{C}$, with the chance of malignancy ranging from $2 \%$ to $95 \%$. Category 5 lesions are highly suspicious and have a greater than $95 \%$ chance of malignancy. Category 6 lesions are biopsy-proven malignant lesions. ${ }^{2}$ This allows for a universal and unambiguous description of various factors in the breast imaging assessment, most importantly by quantifying the degree of suspicion of malignancy of a breast lesion by a single number.

Ultrasound-guided core-needle breast (UGCNB) biopsy is invaluable in the management of patients with breast lesions that require a histological diagnosis to assist with planning further management. UGCNB biopsy has been shown to have many benefits over open surgical biopsy 
in obtaining a histological diagnosis prior to planning definitive management. ${ }^{3,4,5,6}$

Regular audits should be performed within mammography departments in order to assess the radiologists' implementation of the BI-RADS guidelines. This is important to ensure that the unit is performing satisfactorily and adhering to international guidelines. ${ }^{2}$ A review of the literature revealed no published results of such an audit performed at a South African hospital.

This article describes a retrospective analysis of UGCNB biopsies performed at the Grey's Hospital Mammography Department (GHMD). The data collected included the patient demographics, the BI-RADS category, the imaging modalities used to assess the patients and the histological diagnoses obtained from the UGCNB biopsies. The purpose of this study was to perform an audit of the UGCNB biopsies and to analyse the accuracy of the radiologists' use of the BI-RADS scoring system.

\section{Research methods and design}

Records of all patients who underwent UGCNB biopsies at the GHMD between 1 January 2014 and 31 of October 2015 were reviewed. As this was a retrospective, descriptive study, purposive sampling was used.

Records of patients of all ages and both sexes were used in this study, including records where patients had single or multiple lesions biopsied. Records of patients who underwent a core-needle breast biopsy that was not performed under ultrasound guidance were excluded. Further exclusion criteria included fine-needle aspiration, stereotactic coreneedle breast biopsy or axillary lymph node biopsy.

The biopsies were performed by senior registrars and consultants. The experience of the consultants ranged from 0 to 5 years. No consultants had subspeciality qualifications in breast imaging. All BI-RADS classifications were made by consultants.

Data were collected from the Grey's Hospital Radiology Information System (GHRIS) and from the National Health Laboratory Services database. Data were recorded in the form of a spreadsheet using Microsoft Excel. The demographic data collected included the patient's age and sex. The data recorded for each lesion biopsied included the size (maximum dimension on ultrasound), the number of tissue samples obtained from the lesion, the size of the needle used to perform the biopsy, the imaging modality used to assess the lesion (mammography and ultrasound or ultrasound only), the BIRADS category, whether or not the tissue sample was adequate for histological diagnosis and the histological diagnosis.

Data were analysed using Microsoft Excel and STATA v13. Means with standard deviations or medians with interquartile ranges were used to summarise numerical data as appropriate. Descriptive statistics was used to present categorical data as frequencies and percentages.

\section{Ethical considerations}

Ethics approval was obtained from the University of KwaZulu-Natal Biomedical Research Ethics Committee (BE 487/15). Consent was obtained from the Grey's Hospital management.

\section{Results}

A total of 304 UGCNB biopsies were performed on 291 patients. There were 289 female and 2 male patients. The mean age was 49.2 years (standard deviation [s.d.] $=15.9$ ) with a range of 12 years -85 years. The age distribution is shown in Figure 1.

The number of lesions biopsied, the number adequate for histological diagnosis and the most common malignant and benign diagnoses are presented in Figure 2. The histological diagnoses of all the lesions biopsied are listed in Table 1.

In the GHMD, it is routine practice that all patients who have a mammogram also have an ultrasound examination as a combined examination during the same appointment. If required, a UGCNB biopsy is also performed on the same day. Ultrasound as the only modality of investigation is typically performed on patients younger than 30 years, at the referring clinician's discretion and for patients who are unable to tolerate a mammogram. Of the 291 patients included in the study, $82 \%(n=238)$, with a mean age of 53.1 years (s.d. $=13.65)$ and a range of 27 - 85 years, were assessed with ultrasound and mammogram. The remainder of the patients $(n=53,18 \%)$, with a mean age of 31.8 years (s.d. $=$ 13.62) and a range of 12 - 80 years, were assessed using ultrasound imaging only.

Of the BI-RADS category 4 lesions, not all were allocated a subcategory of A, B or C. The data are represented as category 4 'not specified' (NS) along with the subcategories of $\mathrm{A}, \mathrm{B}$ or $\mathrm{C}$. For the lesions assessed using both mammography and ultrasound, only one $(0.4 \%)$ did not have a BI-RADS category included in the report. Thirty-one lesions (58\%) that were assessed with only ultrasound did not have a BI-RADS category recorded. This is illustrated in Figure 3.

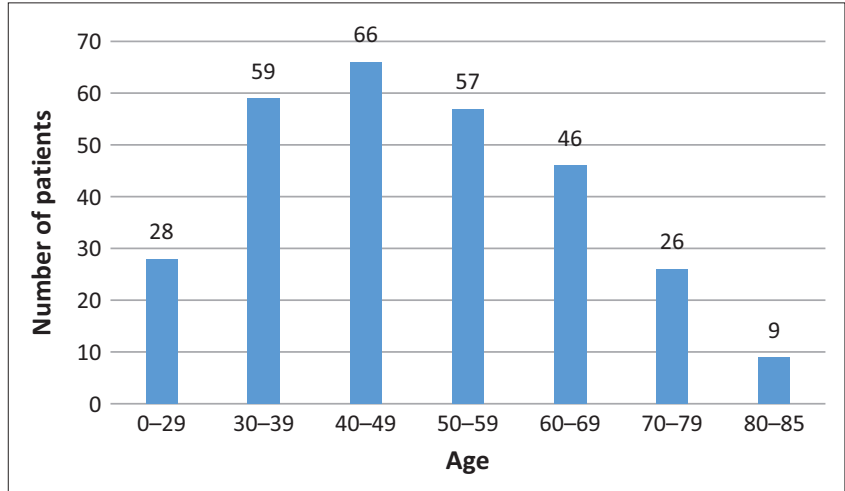

FIGURE 1: Age distribution of the patients in the study $(N=291)$. 


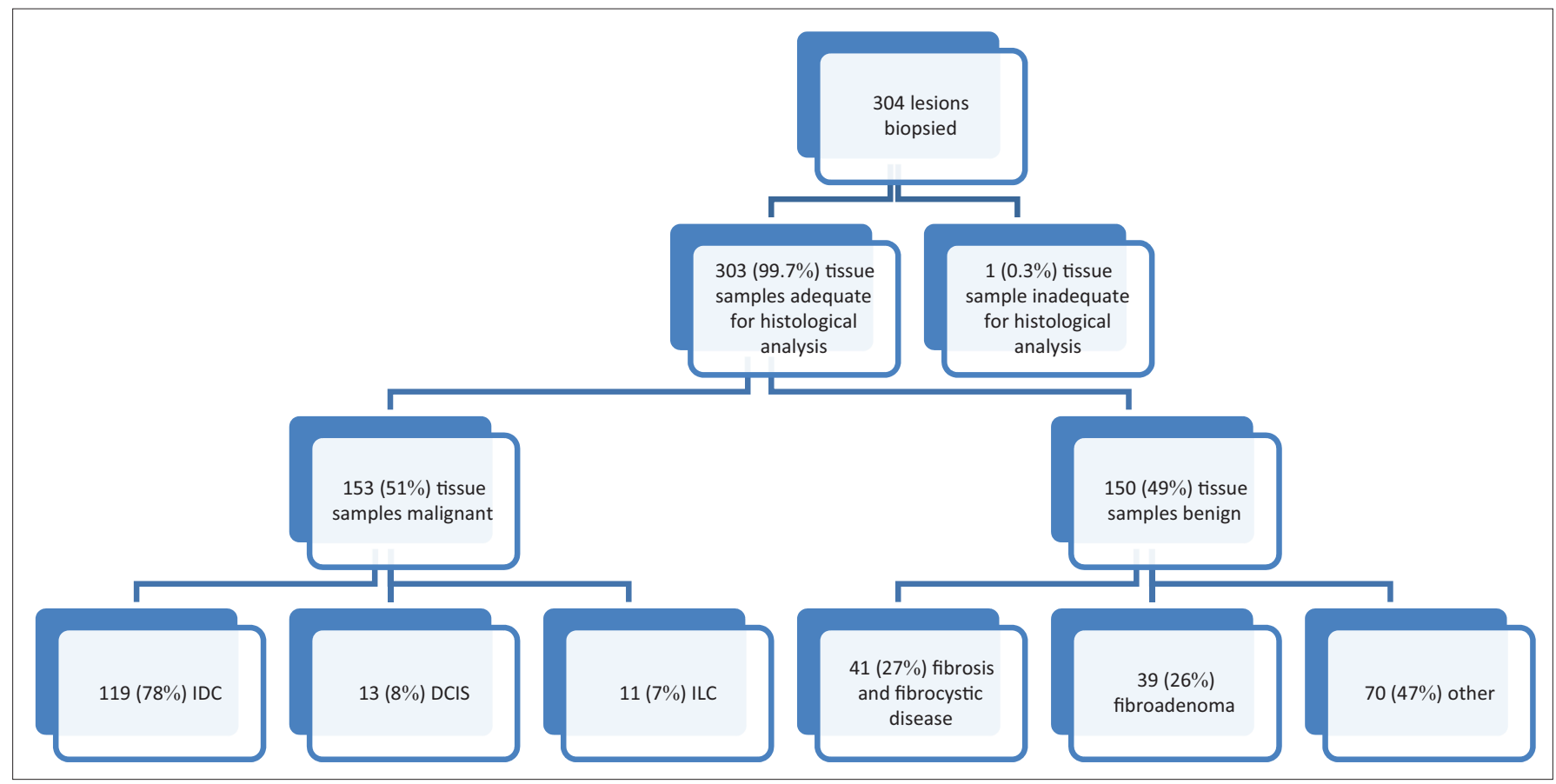

DCIS, ductal carcinoma in situ; IDC, invasive ductal carcinoma.

FIGURE 2: Summary of the results of the lesions biopsied in this study.

TABLE 1: Malignant and benign histological diagnoses of the ultrasound-guided core-needle breast biopsies performed during the study.

\begin{tabular}{lcc}
\hline Histological diagnosis & Number of lesions & $\mathbf{( \% )}$ \\
\hline Malignant & 119 & 78 \\
IDC & 13 & 8 \\
DCIS & 11 & 7 \\
ILC & 2 & 1 \\
Lymphoma & 8 & 6 \\
Malignant other & $\mathbf{1 5 3}$ & $\mathbf{1 0 0}$ \\
\hline Total & & \\
\hline
\end{tabular}

Benign

Fibrosis and fibrocystic

disease

Fibroadenoma

Inflammation/Infection

Fat necrosis

Intraductal papilloma

TB

Benign other

Total

41

27

39

23

22

3

3

19

150

DCIS, ductal carcinoma in situ; IDC, invasive ductal carcinoma; ILC, invasive lobula carcinoma; TB, tuberculosis.

The majority of patients who were evaluated with mammography and ultrasound were assigned BI-RADS category $5(n=112,45 \%)$, and the majority of the patients assessed with ultrasound were assigned equally to BI-RADS category 3 and $4 \mathrm{NS}(n=6,27 \%)$. Tables 2 and 3 list the histology of the lesions in each BI-RADS category according to the imaging modality used. The distribution of the BIRADS categories and the percentage of malignant lesions in each BI-RADS category are shown in Table 4.

A two-by-two table was used to assess the agreement between the imaging features and the histology (Table 5). BI-RADS category 2 and 3 lesions were classed as probably benign or benign imaging findings, whilst category 4 and
5 were classed as possibly malignant imaging findings. There were 30 lesions that were congruent with both benign imaging and histological results. Conversely, 146 out of 237 lesions (62\%) with possibly malignant imaging findings had malignant histology. It is not expected that there would be $100 \%$ concordance as the BI-RADS categories have varying risks of malignancy. It is expected that there would ideally be $100 \%$ concordance only in categories 2 and 6 .

In this study, the BI-RADS classification system as interpreted by the radiologists using the biopsy results as the gold standard had a sensitivity of $98.6 \%$ (95\% confidence interval $(\mathrm{CI})=95 \%-99 \%)$, a specificity of $24.8 \%(95 \% \mathrm{CI}=17 \%-$ $33 \%)$, a positive predictive value (PPV) of $61.6 \%(95 \% \mathrm{CI}=$ $52 \%-68 \%$ ) and a negative predictive value (NPV) of $93.8 \%$ $(95 \% \mathrm{CI}=79 \%-99 \%)$.

The mean size of the breast lesions was $25.8 \mathrm{~mm} \pm 16.8$, with a range of $5 \mathrm{~mm}-100 \mathrm{~mm}$. The size of the lesion was not recorded for $14 \%(n=44)$ of the lesions.

The median number of tissue samples obtained from a lesion was three (mean $=3.14 \pm 0.8$, with a minimum of one tissue sample and a maximum of six tissue samples obtained from a single lesion. In $20 \%(n=62)$ of the lesions, the number of tissue samples obtained was not recorded.

The majority of the lesions $(53 \%, n=147)$ were biopsied using a $14 \mathrm{G}$ needle; an $18 \mathrm{G}$ needle was used for $29 \%(n=82)$ of the lesions and a $16 \mathrm{G}$ needle was used for $18 \%(n=50)$ of the lesions. In $8 \%(n=25)$ of the lesions, the needle size used was not recorded. Of the 29 lesions less than $10 \mathrm{~mm}$ in size, $48 \%(n=14)$ were biopsied using a $14 \mathrm{G}$ needle. 


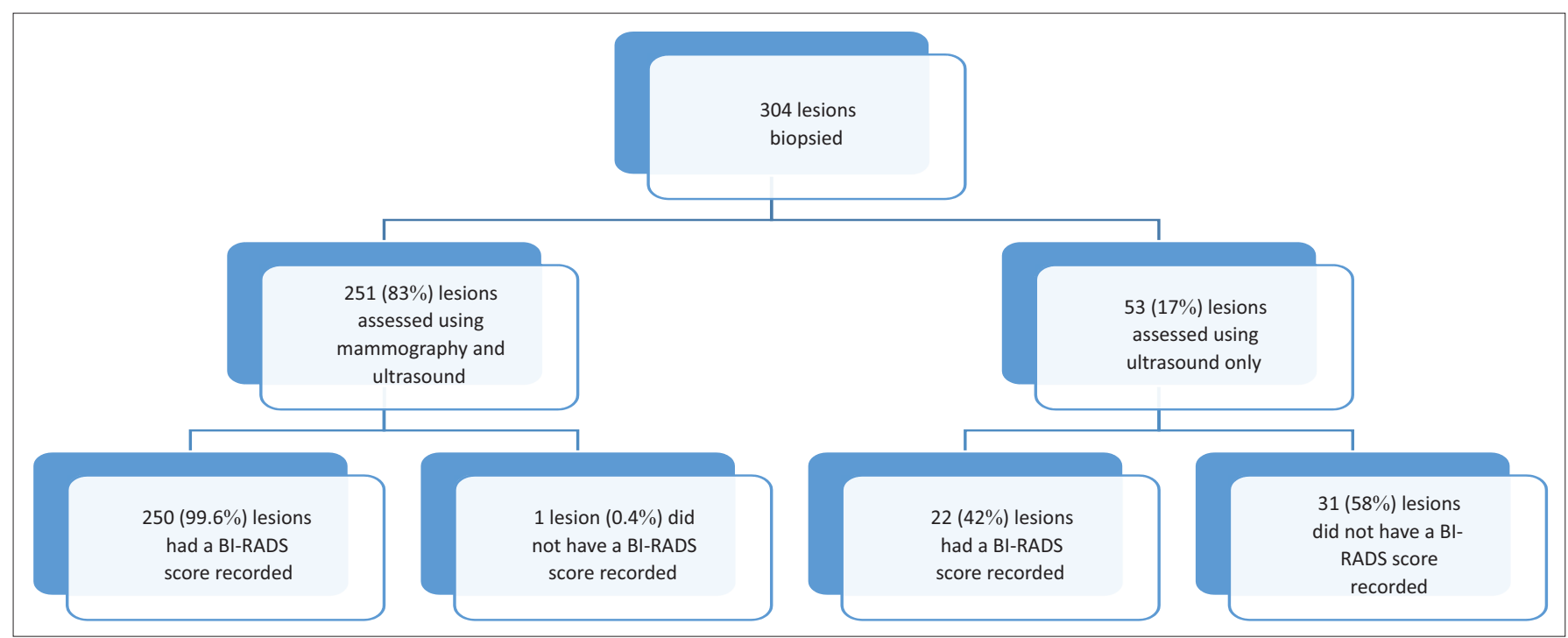

BI-RADS, Breast Imaging Reporting and Data System.

FIGURE 3: Summary of the number of lesions assigned a BI-RADS category.

TABLE 2: Histological diagnoses of the ultrasound-guided core-needle breast biopsy in each BI-RADS category for lesions assessed with mammography and ultrasound.

\begin{tabular}{|c|c|}
\hline BI-RADS category & Number of lesions \\
\hline BI-RADS 2 & 5 \\
\hline Fibroadenoma & 3 \\
\hline Fibrosis and fibrocystic disease & 1 \\
\hline Inflammation/infection & 1 \\
\hline BI-RADS 3 & 19 \\
\hline Fibrosis and fibrocystic disease & 7 \\
\hline Fat necrosis & 3 \\
\hline Inflammation/infection & 3 \\
\hline Fibroadenoma & 2 \\
\hline Benign other & 2 \\
\hline Malignant other & 1 \\
\hline Inadequate sample & 1 \\
\hline BI-RADS 4 NS & 58 \\
\hline IDC & 19 \\
\hline Fibrosis and fibrocystic disease & 11 \\
\hline Fibroadenoma & 8 \\
\hline Fat necrosis & 7 \\
\hline Inflammation/infection & 3 \\
\hline DCIS & 2 \\
\hline TB & 1 \\
\hline Benign other & 5 \\
\hline Malignant other & 2 \\
\hline BI-RADS 4A & 21 \\
\hline Fibroadenoma & 5 \\
\hline Fibrosis and fibrocystic disease & 5 \\
\hline Fat necrosis & 3 \\
\hline Inflammation/infection & 2 \\
\hline Intraductal papilloma & 2 \\
\hline IDC & 1 \\
\hline Benign other & 2 \\
\hline Malignant other & 1 \\
\hline BI-RADS 4B & 14 \\
\hline Fibrosis and fibrocystic disease & 4 \\
\hline IDC & 3 \\
\hline Inflammation/infection & 2 \\
\hline Fat necrosis & 1 \\
\hline Fibroadenoma & 1 \\
\hline
\end{tabular}

Table 2 continues $\rightarrow$
TABLE 2 (Continues...): Histological diagnoses of the ultrasound-guided coreneedle breast biopsy in each BI-RADS category for lesions assessed with mammography and ultrasound.

\begin{tabular}{lc}
\hline BI-RADS category & Number of lesions \\
\hline Intraductal papilloma & 1 \\
Benign other & 1 \\
Malignant other & 1 \\
BI-RADS 4C & 19 \\
IDC & 8 \\
Fibrosis and fibrocystic disease & 4 \\
Fibroadenoma & 3 \\
DCIS & 2 \\
Fat necrosis & 1 \\
Inflammation/infection & 1 \\
BI-RADS 5 & 112 \\
IDC & 80 \\
ILC & 10 \\
DCIS & 9 \\
Fibrosis and fibrocystic disease & 3 \\
Inflammation/infection & 3 \\
Fat necrosis & 2 \\
TB & 1 \\
Lymphoma & 1 \\
Malignant other & 1 \\
BI-RADS 6 & 1 \\
IDC & 3 \\
ILC & 2 \\
BI-RADS not recorded & 1 \\
\hline & 1 \\
\hline
\end{tabular}

BI-RADS, Breast Imaging Reporting and Data System; 4 NS (not specified), BI-RADS category 4 lesions that were not assigned a subcategory of A-C; DCIS, ductal carcinoma in situ; IDC, invasive ductal carcinoma; ILC, invasive lobular carcinoma; TB, tuberculosis.

\section{Discussion}

The GHMD serves a large patient drainage area encompassing 3.9 million (38\%) of KwaZulu-Natal's population. ${ }^{7}$ As mammography is thus a very limited resource, there is no established screening programme and the services provided at the hospital are only diagnostic. Having a standardised guideline such as BI-RADS significantly facilitates this process. In this study, we found that when correlating with UGCNB biopsy, radiologists 
TABLE 3: Histological diagnoses of the ultrasound-guided core-needle breast biopsy in each BI-RADS category for lesions assessed with ultrasound.

\begin{tabular}{|c|c|}
\hline BI-RADS category & Number of lesions \\
\hline BI-RADS 2 & 3 \\
\hline Fibroadenoma & 2 \\
\hline Benign other & 1 \\
\hline BI-RADS 3 & 6 \\
\hline Fibroadenoma & 3 \\
\hline Fibrosis and fibrocystic disease & 1 \\
\hline IDC & 1 \\
\hline Benign other & 1 \\
\hline BI-RADS 4 (NS) & 6 \\
\hline Fat necrosis & 2 \\
\hline Fibrosis and fibrocystic disease & 2 \\
\hline Fibroadenoma & 1 \\
\hline Inflammation/infection & 1 \\
\hline BI-RADS 4A & 2 \\
\hline Fat necrosis & 1 \\
\hline Inflammation/infection & 1 \\
\hline BI-RADS 4B & 1 \\
\hline Inflammation/infection & 1 \\
\hline BI-RAds 4C & 3 \\
\hline IDC & 3 \\
\hline BI-RADS 5 & 1 \\
\hline IDC & 1 \\
\hline BI-RADS not recorded & 31 \\
\hline Fibroadenoma & 11 \\
\hline Inflammation/infection & 5 \\
\hline Fibrosis and fibrocystic disease & 3 \\
\hline Fat necrosis & 2 \\
\hline IDC & 1 \\
\hline Lymphoma & 1 \\
\hline TB & 1 \\
\hline Benign other & 7 \\
\hline
\end{tabular}

BI-RADS, Breast Imaging Reporting and Data System; 4 NS (not specified), BI-RADS category 4 lesions that were not assigned a subcategory of A-C; IDC, invasive ductal carcinoma; TB, tuberculosis.

TABLE 4: Distribution of the number of ultrasound-guided core-needle breast biopsy lesions in each BI-RADS category with a comparison of the percentage of malignant lesions in category with the BI-RADS recommendations.

\begin{tabular}{llll}
\hline BI-RADS category & Number of lesions & $\begin{array}{l}\% \text { of malignant } \\
\text { lesions }\end{array}$ & $\begin{array}{l}\text { BI-RADS recommended } \\
\text { likelihood of cancer }\end{array}$ \\
\hline 2 & 8 & 0 & $0 \%$ \\
3 & 25 & 8 & $>0$ but $\leq 2 \%$ \\
4 NS & 64 & 36 & $>2 \%$ but $<95 \%$ \\
$4 \mathrm{~A}$ & 23 & 9 & $>2$ but $\leq 10 \%$ \\
$4 \mathrm{~B}$ & 15 & 27 & $>10 \%$ but $\leq 50 \%$ \\
$4 \mathrm{C}$ & 22 & 59 & $>50 \%$ but $<95 \%$ \\
5 & 113 & 92 & $\geq 95 \%$ \\
6 & 2 & 100 & $\begin{array}{l}\text { Biopsy-proven } \\
\text { malignancy }\end{array}$ \\
Not recorded & 32 & 9 & - \\
\hline
\end{tabular}

Note: 4 NS (not specified), BI-RADS category 4 lesions that were not assigned a subcategory of A-C.

TABLE 5: Two-by-two table comparing the imaging assessment and histology results.

\begin{tabular}{lccc}
\hline Imaging assessment & \multicolumn{2}{c}{ Lesion histology } & Total \\
\cline { 2 - 3 } & Benign & Malignant & \\
\hline Probably benign or benign & 30 & 2 & 32 \\
Possibly malignant & 91 & 146 & 237 \\
Total & $\mathbf{1 2 1}$ & $\mathbf{1 4 8}$ & $\mathbf{2 6 9}$ \\
\hline
\end{tabular}

BI-RADS category 2 and 3 lesions were grouped as probably benign or benign and BI-RADS category 4 and 5 lesions were grouped as possibly malignant.

Note: Of the 272 lesions that were assigned a BI-RADS category, 269 lesions are included in this table; the two category 6 lesions and the one lesion that was not suitable for histological diagnosis are excluded. interpreted the BI-RADS score with a high sensitivity of $98.6 \%$ but with a lower specificity of $24.8 \%$.

The overall PPV of $61.6 \%$ in this study is much higher than the $35.9 \%$ stipulated by the BI-RADS guidelines for diagnostic mammography. ${ }^{2}$ This could be explained by the long waiting times to access specialised breast imaging services, resulting in a higher proportion of malignant lesions being biopsied. Another consideration is that the BI-RADS guidelines do not have set values for combined mammography and ultrasound evaluation and thus the results may differ from the diagnostic mammography guidelines. ${ }^{2}$ The PPV of the BI-RADS categories was, however, within the limits stipulated by the guidelines for categories 2, 4A, 4B and 4C (Table 4). The NPV of $93.8 \%$ in this study is based on the biopsy results. In a study which analysed the NPV of UGCNB biopsies, it was found to be $99.4 \% .{ }^{8}$ The BI-RADS guidelines do not stipulate an NPV.

According to the guidelines, category 2 lesions require routine screening and category 3 lesions should undergo short interval follow-up. ${ }^{2}$ Given the limited access to specialised care, the recommended practice is often altered, whereby lesions with benign features are biopsied upon patient request as opposed to only being followed up by imaging. As a result of this, we biopsied a higher proportion of category 2 BI-RADS lesions (4\%) in comparison with a previous study by Wiratkapun et $\mathrm{al}^{3}(2 \%)$.

The guidelines also note that the risk of malignancy for category 3 lesions is less than or equal to $2 \% .^{2}$ In this study, 2 out of 25 patients (8\%) with lesions categorised as BI-RADS 3 had malignant histology. The first was a patient with a 6-month history of a breast lump that had imaging features suggestive of a papilloma with histology demonstrating a papillary carcinoma. The second patient had previously undergone a unilateral mastectomy and whilst on surveillance was found to have an intramammary lymph node that had increased in size. Histology demonstrated invasive ductal carcinoma (IDC). This lesion was incorrectly categorised as BI-RADS 3.

High-risk lesions (Box 1) are histological diagnoses on core biopsy that imply a high-risk of developing malignancy. In addition, excision biopsies performed on these lesions often result in malignant histological findings.,9 Papillomas are controversial lesions, with some authors classifying these as high-risk lesions; however, in this study, we followed the approach of Wiratkapun et al. ${ }^{3}$ and Youk et al. ${ }^{9}$ who consider these as benign lesions. Thus, in this study, there were no high-risk lesions in contrast to other similar studies. ${ }^{3,9}$ An explanation for this may be that the patients assessed in the unit are all symptomatic as no screening service is offered. The long waiting time may also have resulted in malignancy having already developed by the time the biopsy was performed. 
BOX 1: High-risk histological diagnoses.

\begin{tabular}{l|}
\hline Atypical ductal hyperplasia \\
Lobular neoplasia \\
Radial sclerosing lesion \\
Papillary lesion with atypical ductal hyperplasia \\
Phyllodes tumour \\
\hline
\end{tabular}

Source: Adapted from: Wiratkapun C, Treesit T, Wibulpolprasert B, Lertsithichai P. Diagnostic accuracy of ultrasonography-guided core needle biopsy for breast lesions. Singapore Med J. 2012;53(1):40-45; G, Schueller-Weidekamm C, Helbich T. Accuracy of ultrasound-guided large-core needle breast biopsy. Eur Radiol. 2008:18:1761-1773. https://doi.org/10.1007/ large-core s00330-008-0955-4; Schueller G, Jaromi S, Ponhold L, et al. US-guided 14-gauge core-needle breast biopsy: Results of a validation study in 1352 cases. Radiology. 2008;248:406-413 https://doi.org/10.1148/radiol.2482071994; Youk JH, Kim KE, Kim MJ, Oh KK. Sonographically guided 14-gauge core needle biopsy of breast masses: A review of 2,420 cases with long term follow-up. AJR Am J Roentgenol. 2008;190:202-207. https://doi.org/10.2214/ AJR.07.2419

Note: These are histological diagnoses on UGCNB biopsy associated with a high rate of underestimation compared to the final diagnosis.

In one interesting case, a patient with a strong family history of breast cancer with a 3-year history of bilateral breast lumps (Figure 4) underwent bilateral UGCNB biopsies. The biopsies demonstrated IDC in the right breast and invasive lobular carcinoma in the left breast. This histology was confirmed on examination of the mastectomy specimens. The incidence of synchronous bilateral breast carcinoma amongst breast cancer patients was found to be $0.8 \%$ in a study by Díaz et al. ${ }^{10}$ Furthermore, our case is of particular interest because in a study by Gong et al., ${ }^{11}$ 93\% of patients with synchronous breast cancer had the same histological type.

At GHMD, the routine use of diagnostic mammography is usually limited to patients over the age of 30 . There were two patients under the age of 30 on whom mammograms were performed; the decision to perform these mammograms was based on the clinical history and suspicious features of the lesion on ultrasound imaging. The histology for both patients was benign, with one lesion demonstrating inflammatory changes and the other fat necrosis; these lesions were assigned BI-RADS category $4 \mathrm{~B}$ and $4 \mathrm{NS}$, respectively. The patient with inflammatory changes had imaging features thought to represent either atypical fibroadenomas or malignancy. The histology from the UGCNB biopsy was not congruent with the imaging findings and an excision biopsy should have been considered. ${ }^{5,6}$

There were eight patients with a histological result of 'benign breast tissue'. Of these patients, one had accessory breast tissue and one had oedema underlying a skin lesion which resolved. Of the remaining six patients, three were followed up with benign findings confirmed on imaging in two of the patients and repeat biopsy in the third. The remaining three patients were not followed up, despite the discordant imaging and histology results. Of these, two patients had imaging features of fibroadenomas and one patient had an intraductal lesion.

The preceding cases illustrate a known limitation of UGCNB biopsy, namely false-negative results. The false-negative rate in the literature ranges from $0.1 \%$ to $4 \% .{ }^{3,5,6,8,9}$ Underestimation of the disease process, another limitation of UGCNB biopsy, has been shown to be most common for ductal carcinoma in
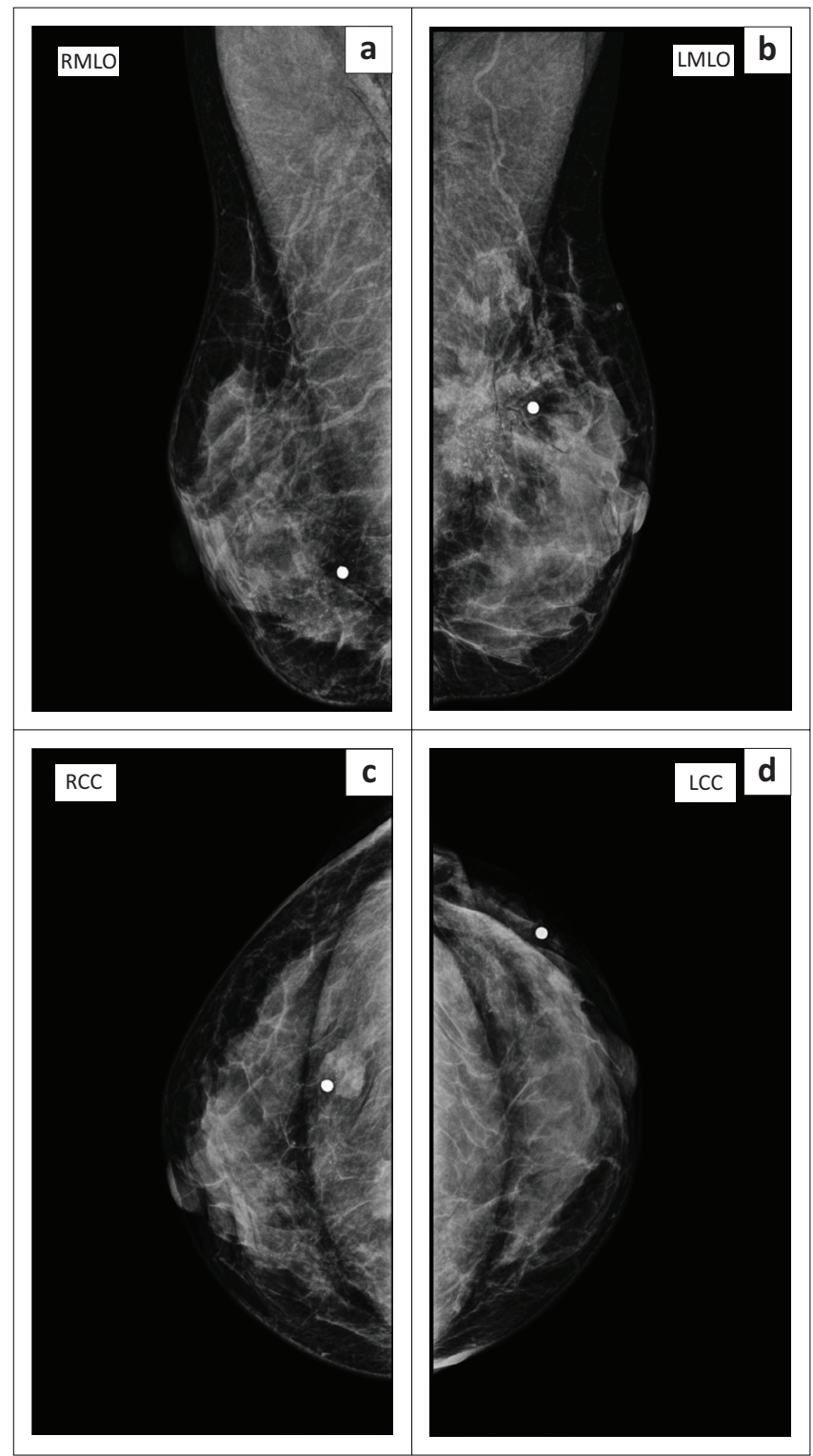

CC, craniocaudal; IDC, invasive ductal carcinoma; ILC, invasive lobular carcinoma; MLO, mediolateral oblique.

FIGURE 4: The right MLO (a), left MLO (b), right CC (c) and left CC (d) mammogram images of a 38-year-old woman who presented with a 3-year history of bilateral breast lumps. The skin markers demonstrate the site of the palpable lesion in each breast. In the central lower quadrant of the right breast, a focal soft tissue density is present with associated pleomorphic calcifications . In the left breast, there is a larger soft tissue density with spiculated margins, architectural distortion and pleomorphic calcifications in the upper outer quadrant. Both lesions were hypoechoic on ultrasound, with poorly defined margins and posterior shadowing. Ultrasound-guided core-needle biopsy demonstrated IDC in the right breast and ILC in the left breast; this histology was confirmed after mastectomy.

situ, lobular carcinoma in situ and atypical ductal hyperplasia. ${ }^{3}$ The underestimation rate of UGCNB was shown to be $31.4 \%$ in a previous study. ${ }^{5}$

A previous study has advocated that $14 \mathrm{G}$ needles should be used in all lesions less than $1 \mathrm{~cm}$ in size, with $16 \mathrm{G}$ and $18 \mathrm{G}$ having comparable results with $14 \mathrm{G}$ needles in lesions greater than $1 \mathrm{~cm}$ in size, ${ }^{12}$ whereas a different study has shown that $14 \mathrm{G}$ needles are more accurate, and have a decreased underestimation and false-negative rate when compared with $16 \mathrm{G}$ needles. ${ }^{13}$ In the GHMD, this is difficult to adhere to because of stock shortages often resulting in only one size of 
needle being available. In our study, there was no significant correlation found between lesion size and needle size.

Other studies have included the number of tissue samples obtained..$^{8,13}$ The mean number of $3.14 \pm 0.8$ tissue samples in this study is similar to previous studies that demonstrated means of 3.98 and 3.0, respectively. ${ }^{8,13}$ A study which investigated the optimal number of core tissue samples needed to obtain a diagnosis demonstrated a $98 \%$ diagnostic yield when three core samples were obtained and $100 \%$ diagnostic yield when five core samples were obtained. ${ }^{14}$ In our study, the diagnostic yield was $99.7 \%$ (303 out of 304 lesions).

Tuberculosis (TB) of the breast has a reported prevalence of $3 \%-4.5 \%$ in all surgical breast lesions in endemic countries. ${ }^{15}$ A recent study performed in South Africa demonstrated a prevalence of $0.3 \%$ in all patients who had undergone breast investigation. ${ }^{15}$ In this study, three $(1 \%)$ patients had confirmed TB. This may be an underestimation of the true prevalence of the disease in our population as the diagnosis may have been made at a peripheral hospital. This recent study also showed that the yield of acid-fast bacilli on histology specimens which demonstrated necrotising granulomatous inflammation was only $29.6 \%{ }^{15}$ It is thus possible that some patients in our study could have been misdiagnosed as necrotising granulomatous inflammation if a TB culture was not performed.

\section{Limitations of the study}

The sensitivity and specificity in this study should be interpreted with caution as these were derived from the biopsy results and not from a tumour registry or long-term follow-up as per the BI-RADS guidelines. Likewise, the falsenegative rate in this study could not be evaluated as the above data were not available.

GHMD only offers a diagnostic service. This limits comparability with other studies in mammography departments which offer a screening programme.

\section{Recommendations}

The findings in this study have highlighted some of the shortcomings of the GHMD service. The findings will be used to alter and improve departmental practice. One such shortcoming was that patients who were assessed with only ultrasound did not always have a BI-RADS category assigned to them, and the patients assessed as BI-RADS category 4 were not further stratified into subgroups A-C. The addition of this information to the generic template on the GHRIS system will assist with correcting this.

Radiologists will be encouraged to review the UGCNB results and for patients with discordant lesions to be flagged, so that further management can be instituted. This practice would be beneficial to registrar training and will improve patient care. Discussion of these lesions at the regular interdisciplinary meeting will be advised.
Double reading of studies and ongoing training could be implemented to decrease radiologist error and ensure better compliance with the BI-RADS guidelines.

\section{Conclusion}

The study describes the patient and lesion profile and unit practices in a tertiary hospital setting in South Africa. The radiologists' application of the BI-RADS scoring largely conforms to the BI-RADS guidelines. However, the study highlights several challenges encountered by a breast imaging programme in an under-resourced setting, as well as making recommendations for overcoming these challenges. The lack of screening service, large patient load and combined mammography and ultrasound assessments are some of the challenges faced and practices implemented in a developing country setting.

\section{Acknowledgements}

The authors thank Catherine Connolly for assistance with the statistical analysis of the data.

\section{Competing interests}

The authors declare that they have no financial or personal relationships that may have inappropriately influenced them in writing this article.

\section{Authors' contributions}

C.A.S. was the main author and was responsible for the protocol, data collection and compilation of the manuscript. M.D.M.G. was the supervisor and assisted with project design, review and analysis. C.A.S. was the principal investigator and prepared the manuscript. M.D.M.G. made conceptual contributions and is the supervisor of C.A.S.'s Master's degree.

\section{References}

1. Summary statistics of cancer diagnosed histologically in South Africa in 2011 [homepage on the Internet]. No date [cited 2016 Oct 31]. Available from: http:// www.cansa.org.za/files/2016/08/NCR-2011-cancer-tables.pdf

2. American College of Radiology. ACR BI-RADS Atlas [homepage on the internet]. 5th ed. c2013 [cited 2016 Oct 31]. Available from: http://www.acr.org/QualitySafety/Resources/BIRADS

3. Wiratkapun C, Treesit T, Wibulpolprasert B, Lertsithichai P. Diagnostic accuracy of ultrasonography-guided core needle biopsy for breast lesions. Singapore Med J. 2012;53(1):40-45.

4. Schueller G, Schueller-Weidekamm C, Helbich T. Accuracy of ultrasound-guided, large-core needle breast biopsy. Eur Radiol. 2008:18:1761-1773. https://doi. org/10.1007/s00330-008-0955-4

5. Schueller G, Jaromi S, Ponhold L, et al. US-guided 14-gauge core-needle breast biopsy: Results of a validation study in 1352 cases. Radiology. 2008;248:406-413. https://doi.org/10.1148/radiol.2482071994

6. Youk JH, Kim E-K, Kim MJ, Lee JY, Oh KK. Missed breast cancers at US-guided core needle biopsy: How to reduce them. Radiographics. 2007;27:79-94. https://doi. org/10.1148/rg.271065029

7. Frith A. Census 2011 [homepage on the Internet]. No date [cited 2016 Oct 30]. Available from: https://census2011.adrianfrith.com/place/5

8. Zhang C, Lewis DR, Nasute P, Hayes M, Warren L, Gordon PB. The negative predictive value of ultrasound-guided 14-gauge core needle biopsy of breast masses: A validation study of 339 cases. Cancer Imaging. 2012;12:488-496. https://doi.org/10.1102/1470-7330.2012.0047

9. Youk JH, Kim KE, Kim MJ, Oh KK. Sonographically guided 14-gauge core needle biopsy of breast masses: A review of 2,420 cases with long-term follow-up. AJR Am J Roentgenol. 2008;190:202-207. https://doi.org/10.2214/AJR.07.2419 
10. Díaz R, Munárriz B, Santaballa A, et al. Synchronous and metachronous bilateral breast cancer: A long-term single-institution experience. Med Oncol. 2012;29(1):16-24. https://doi.org/10.1007/s12032-010-9785-8

11. Gong SJ, Rha SY, Jeung HC, Roh JK, Yang WI, Chung HC. Bilateral breast cancer: Differential diagnosis using histological and biological parameters. Jpn I Clin Oncol. 2007;37(7):487-492. https://doi.org/10.1093/jjco/hym056

12. Zhou J-Y, Tang J, Wang Z-L, et al. Accuracy of 16/18G core needle biopsy for ultrasound-visible breast lesions. World J Surg Oncol. 2014;12:7. https://doi. org/10.1186/1477-7819-12-7
13. Lai HW, Wu HK, Kuo SJ, et al. Differences in accuracy and underestimation rates for 14 - versus 16-gauge core needle biopsies in ultrasound-detectable breast lesions. Asian J Surg. 2013;36(2):83-88. https://doi.org/10.1016/j.asjsur.2012.09.003

14. Kirshenbaum K, Keppke A, Hou K, Dickerson M, Gajjar M, Kirshenbaum G. Reassessing specimen number and diagnostic yield of ultrasound guided breast core biopsy. Breast J. 2012;18(5):464-469. https://doi.org/10.1111/j.1524-4741.2012.01269.x

15. Ramaema DP, Buccimazza I, Hift RJ. Prevalence of breast tuberculosis: Retrospective analysis of 65 patients attending a tertiary hospital in Durban, South Africa. S Afr Med J. 2015;105(10):866-869. https://doi.org/10.7196/SAMJnew.7704 